\title{
Carbon C 14 Gilteritinib
}

National Cancer Institute

\section{Source}

National Cancer Institute. Carbon C 14 Gilteritinib. NCI Thesaurus. Code C122677.

A radioconjug ate composed of gilteritinib, an inhibitor of the receptor tyrosine kinases (RT Ks) FMS-related tyrosine kinase 3 (FLT3, ST K1, or FLK2), AXL (UFO or JT K11) and anaplastic lymphoma kinase (ALK or CD246), labeled with the radioisotope carbon C 14, with potential use for evaluating the pharmacokinetic profile of gilteritinib. Gilteritinib binds to and inhibits both the wild-type and mutated forms of FLT3, AXL and ALK. This may result in an inhibition of FLT3, AXL, and ALK-mediated signal transduction pathways and the reduction of tumor cell proliferation in cancers that overexpress these RT Ks. Labeling of gilteritinib with the radioactive tracer carbon C 14 allows for the evaluation of gilteritinib's pharmacokinetic profile, including its absorption, distribution, metabolism, and excretion (ADME). 